\title{
Getting the most neutrinos out of IsoDAR
}

\author{
Emilio Ciuffoli $^{1, \mathrm{a}}$, Hosam Mohammed ${ }^{1,2, \mathrm{~b}}$, Jarah Evslin ${ }^{1,2, \mathrm{c}}$, Fengyi Zhao ${ }^{1, \mathrm{~d}}$, Maksym Deliyergiyev ${ }^{1, \mathrm{e}}$ \\ ${ }^{1}$ Institute of Modern Physics, CAS, NanChangLu 509, Lanzhou 730000, China \\ ${ }^{2}$ University of the Chinese Academy of Sciences, YuQuanLu 19A, Beijing 100049, China
}

Received: 13 July 2016 / Accepted: 4 December 2017 / Published online: 13 December 2017

(C) The Author(s) 2017. This article is an open access publication

\begin{abstract}
Several experimental collaborations worldwide intend to test sterile neutrino models by measuring the disappearance of antineutrinos produced via isotope decay at rest (IsoDAR). The most advanced of these proposals have very similar setups, in which a proton beam strikes a target yielding neutrons which are absorbed by a high isotopic purity ${ }^{7} \mathrm{Li}$ converter, yielding ${ }^{8} \mathrm{Li}$ whose resulting decay yields the antineutrinos. In this note, we use FLUKA and GEANT4 simulations to investigate three proposed modifications of this standard proposal. In the first, the ${ }^{7} \mathrm{Li}$ is replaced with ${ }^{7} \mathrm{Li}$ compounds including a deuterium moderator. In the second, a gap is placed between the target and the converter to reduce the neutron bounce-back. Finally, we consider cooling the converter with liquid nitrogen. We find that these modifications can increase the antineutrino yield by as much as $50 \%$. The first also substantially reduces the quantity of high purity ${ }^{7} \mathrm{Li}$ which is needed.
\end{abstract}

\section{Introduction}

\subsection{Motivation}

Various anomalies can be explained if one invokes sterile neutrinos. Among the most intriguing of these is the LSND anomaly [1,2], in which $\bar{v}_{e}$ appeared in a detector located $30 \mathrm{~m}$ away from a $\mu^{+}$decay at rest $\bar{v}_{\mu}$ source. While the appearance signal is in nearly $4 \sigma$ of tension with a three flavor mixing model, it is easily explained in the presence of a single flavor of sterile neutrino. However other appearance experiments in the same channel have produced negative [3, 4] or inconclusive [5] results. Anomalous disappearance of $\bar{v}_{e}$

\footnotetext{
a e-mail: ciuffoli@impcas.ac.cn

b e-mail: hosam@impcas.ac.cn

ce-mail: jarah@impcas.ac.cn

de-mail: fengchu@impcas.ac.cn

e e-mail: deliyergiyev@impcas.ac.cn
}

produced by a radioactive source in a detector [6,7], called the Gallium anomaly, can also be explained with a single flavor of sterile neutrino with a mass of at least $1 \mathrm{eV}$.

The deficit of measured reactor antineutrinos with respect to state-of-the-art predictions $[8,9]$, called the reactor anomaly [10], can also be explained with a single sterile neutrino with a mass which may be as small as $0.1 \mathrm{eV}$. However in this case the shape of the observed spectrum [11-14] is in disagreement with the theoretical predictions. It is more difficult for sterile neutrinos to explain this spectral deformation, and so many authors have instead argued that it results from a fault in the calculations of the theoretical spectra $[15,16]$. Consistent with this interpretation of the deficit is recent evidence from Daya Bay that the contributions of the primary fission isotopes to the deficit are not proportional to their abundances [17].

Such massive sterile neutrinos are in some tension with Planck CMB data [18]. However, assuming the standard $\Lambda \mathrm{CDM}$ cosmological model, the Planck data is in $3 \sigma$ of tension with an every growing list of measurements, from Lyman $\alpha$ forest baryon acoustic oscillations [19] to the Hubble constant as determined by the local distance ladder [20]. While massive sterile neutrinos alone cannot eliminate these tensions [21,22], they do ease the tension by increasing the uncertainties reported by Planck [18] and they may be part of a larger solution involving dynamical dark energy [23-25].

\subsection{Sterile neutrino searches}

Often motivated by these anomalies, there have been a number of proposed experiments which will search for sterile neutrinos. Most of these proposals search for sterile neutrinos in the disappearance channel $\bar{v}_{e} \rightarrow \bar{v}_{e}$. Experiments using reactor neutrinos are at an advanced stage [26]: some have completed runs [27], are already running [28-30], have a running prototype detector module [31], or are under construction [32]. 
As explained in Ref. [33], $\beta$ decay experiments are sensitive to sterile neutrino oscillations if the sterile neutrino is sufficiently massive. In Ref. [34], the authors used the $\beta$ decay experiments Mainz [35] and Troitsk [36], fit together with $\nu_{e}$ and $\bar{v}_{e}$ disappearance data, to derive a $2 \sigma$ upper bound on the sterile neutrino mass squared splitting of $29 \mathrm{eV}^{2}$.

CPT invariance implies that the $v_{e}$ and $\bar{v}_{e}$ survival probabilities are equal, as are those of $\nu_{\mu}$ and $\bar{\nu}_{\mu}$. If furthermore there is only a single flavor of sterile neutrino, then the $v_{e}$ and $v_{\mu}$ survival probabilities as well as the $v_{e} \leftrightarrow v_{\mu}$ transition rates are determined by only two parameters, $U_{e 4}$ and $U_{\mu 4}$. This implies that anomalies in the $\bar{v}_{e}$ appearance and disappearance channels, when combined, place constraints on $v_{\mu}$ disappearance and vice versa. So far there is no evidence for $v_{\mu}$ disappearance at shorter baselines than would be expected from the standard mixing of the 3 active neutrinos. This lack of evidence therefore constrains $\bar{\nu}_{e}$ appearance and disappearance due to a single flavor of sterile neutrino. In particular, Ref. [34] has shown that recent limits on short baseline $v_{\mu}$ disappearance by MINOS [37] and IceCube [38], when incorporated into a global fit, exclude sterile neutrino mass squared splittings below $1 \mathrm{eV}^{2}$ at the $2 \sigma$ level.

According to Ref. [34], in a large part of the remaining parameter space, disappearance channel sterile neutrino searches using Isotope Decay At Rest (IsoDAR) are particularly sensitive. To our knowledge in all such proposals a neutron source produces neutrons which are absorbed by ${ }^{7} \mathrm{Li}$. This produces ${ }^{8} \mathrm{Li}$ whose decay produces $\bar{v}_{e}$ with a well known energy spectrum, extending to $13 \mathrm{MeV}$, with an average energy of $6.5 \mathrm{MeV}$. The neutron source is usually a high intensity accelerator [39-41], but can also be an intense neutron emitting isotope [42] or a nuclear reactor [43]. This canonical setup was first proposed in Ref. [44], in which the neutron source was a "special nuclear reactor". After half a century, this idea has come full circle with proposals to use an accelerator driven system (ADS) subcritical reactor as the neutron source.

The most advanced proposals have been made by the DAE $\delta$ ALUS collaboration. The neutron sources in these proposals are cyclotrons which are under development as part of an ADS reactor and active interrogation program [39]. The cyclotrons accelerate a high intensity proton beam or a $\mathrm{H}_{2}^{+}$ beam which is then dissociated into a proton beam. The proton beam energy is $60 \mathrm{MeV}$ with a current of $10 \mathrm{~mA}$. The protons strike a beryllium target, creating spallation neutrons. In some cases the neutrons exit from the target into a heavy water moderator $[45,46]$, while in some cases there is no moderator [47]. In either case, they then enter into a sleeve containing isotopically pure ${ }^{7} \mathrm{Li}$, sometimes in the compound FLiBe $\left(\mathrm{Li}_{2} \mathrm{BeF}_{4}\right)$ [47], where they are absorbed yielding ${ }^{8} \mathrm{Li}$. The eventual ${ }^{8} \mathrm{Li}$ decay creates $\bar{\nu}_{e}$ with a well-known energy spectrum [48-50].
IsoDAR disappearance channel experiments have several advantages over reactor neutrino experiments. First, the fact that the spectrum is fairly well known reduces a major source of error in reactor neutrino experiments. Second, after weighting by the inverse $\beta$ decay cross section, $87 \%$ of the $\bar{\nu}_{e}$ have energies above $6 \mathrm{MeV}$. At these relatively high energies the accidental background that plagues reactor experiments is reduced by two orders of magnitude [28]. Third, this higher energy means that the same distance to energy ratio $L / E$, and so the same oscillation phase, is achieved at greater distances. As a result more shielding can be added and, more crucially, distance resolution requirements are weakened. This also allows access to higher sterile neutrino masses.

To save money, all IsoDAR proposals use existing infrastructure, either the accelerator or the detector. In particular DAE $\delta$ ALUS has provided a detailed proposal for an IsoDAR experiment at KamLAND [47] and the JUNO collaboration has also included such an experiment in its plan [46]. In addition in Refs. [40,51] IsoDAR experiments have been proposed using the LINACs that are being built for China's Accelerator Driven System (ADS) subcritical reactor project. In particular, a $25 \mathrm{MeV}, 10 \mathrm{~mA}$ proton accelerator will be completed this year, although for now perhaps only at $5 \mathrm{~mA}$, and a 250-600 MeV, $10 \mathrm{~mA}$ accelerator called China Initial ADS (CI-ADS) will be completed in 2022, with civil engineering beginning this year.

\subsection{Summary of results}

The target stations of all four of the above IsoDAR proposals are quite similar. In this paper we will present the results of our simulations of various modifications of these target stations. Our objective is to determine to what extent various modifications affect the $\bar{v}_{e}$ yield. In particular we will not be interested here in how these modifications may be implemented, in the effect on the sensitivity to sterile neutrino searches or other science goals or even on the absolute normalization of the $\bar{v}_{e}$ flux. As a result our study is quite straightforward, lending itself to simulation with FLUKA and also with GEANT4 using the physics list FTFP_BERT_HP below $250 \mathrm{MeV}$ and QGSP_BIC_HP at $250 \mathrm{MeV}$.

These results will lead us to three main conclusions:

1. Mixing the moderator and the ${ }^{7} \mathrm{Li}$ converter [52] increases the $\bar{v}_{e}$ yield by as much as $50 \%$.

2. In this mixed case, for a sufficiently large converter, the $\bar{\nu}_{e}$ rate can be estimated quite precisely using only the overall normalization of the neutron yield, the cross sections and a simple analytic formula.

3. In the case of the $250 \mathrm{MeV}$ CI-ADS beam with the preferred $W$ target, a gap between the target and the con- 
verter can reduce the neutrons lost by bounce-back into the target by as much as $30 \%$.

We will organize our discussion by dividing the simulation into parts. First, in Sect. 2 we will describe the transport of neutrons in the converter and the resulting isotope production. This will be done in an idealized setting with no proton beam or target and a monochromatic neutron source. In Sect. 3 we will consider the proton beam striking the target and the production of spallation neutrons. Finally in Sect. 4 we will describe our full simulations, from the proton beam to ${ }^{8} \mathrm{Li}$ production.

\section{Neutron transport}

In this section we will consider the simpler problem of calculating the $\bar{v}_{e}$ rate given a monochromatic neutron source in the center of the converter which is surrounded by a graphite reflector. We will see that at the relevant energies, the $\bar{v}_{e}$ yield has little dependence on the neutron energy and, for a large enough converter, can easily be estimated analytically. We feel that the results in this section provide an intuitive understanding of the results of the full target station simulations which will be presented in Sect. 4.

\subsection{Analytic results}

In this subsection we will make the crude approximation that the converter is infinite in extent and try to anticipate the expected behavior of the neutrons in a simplified random walk model. All of the converters which we will consider consist of $\mathrm{H}, \mathrm{D}, \mathrm{Be},{ }^{6} \mathrm{Li},{ }^{7} \mathrm{Li}, \mathrm{O}$ and $\mathrm{F}$, with $\mathrm{O}$ having the isotopic abundances found in nature. At the energies of interest the absorption cross sections are inversely proportional to the neutron velocity, and the elastic scattering cross sections are energy-independent up to about $1 \mathrm{MeV}$, where they begin to fall. Above $100 \mathrm{keV}$ there are also some resonances which affect these cross sections considerably. These resonances will not be included in our analytical model, although of course they are incorporated into the libraries used by our simulations.

The vast majority of our neutrons will have initial energies between $100 \mathrm{keV}$ and $5 \mathrm{MeV}$, and so we approximate the elastic scattering cross sections to be energy-independent. We will use the cross sections from Ref. [53] which are summarized in Table 1. All absorption cross sections are reported at $0.025 \mathrm{eV}$, to convert to other energies it suffices to scale inversely by the mean neutron velocity.

Let $\rho_{i}$ be the number of isotopes of type $i$ per unit volume and let $\sigma_{\text {elastic }}^{i}$ be the elastic scattering cross section for a neutron on an isotope of type $i$. Considering only elastic scattering, as is reasonable before the neutrons are thermalized, the mean free path is $\lambda=\frac{1}{\sum_{i} \rho_{i} \sigma^{i}}$.

We will consider a simple model of neutron moderation in which the neutron loses $40 \%$ of its energy during each collision with a $\mathrm{D}$, and no energy during the other collisions. In particular we will ignore elastic scattering with $\mathrm{H}$ which will be quite rare in the cases that follow as a result of the high isotopic purity of $\mathrm{D}$. The probability that a given elastic scattering involves a $\mathrm{D}$ is equal to

$p=\rho_{D} \sigma^{D} \lambda$

Therefore roughly every $1 / p$ elastic scatterings there is an elastic scattering with a D. Let us assume that at each of these $1 / p$ scatterings, as the target is much heavier than the neutron, the neutron's direction is randomized and so the neutron follows a 3-dimensional random walk. Therefore the neutron will travel, on average, a distance

$d_{D}=\sqrt{\frac{2}{3 \pi p} \lambda}=\sqrt{\frac{2 \lambda}{3 \pi \rho_{D} \sigma^{D}}}$

in any given direction between two elastic scatterings with D.

A neutron which is created with an energy of $E \mathrm{MeV}$ will thermalize to room temperature after $-\ln \left(4 E \times 10^{7}\right) / \ln (0.6)$ collisions with $\mathrm{D}$. Now we will make the poor approximation that the neutron randomizes its direction when scattering with $\mathrm{D}$, effectively ignoring the recoil of the deuteron. Then during these collisions, it will travel an expected distance of

$d_{\text {therm }}=\sqrt{\frac{2 \lambda \ln \left(4 E \times 10^{7}\right)}{3 \pi \ln (5 / 3) \rho_{D} \sigma^{D}}}$

in a given direction. For example, one expects neutrons to travel a distance $d_{\text {therm }}$ in the radial direction of a wide, hollow, cylindrical converter before thermalization. We have numerically simulated random walks with and without $\mathrm{D}$ recoil, assuming that the converter consists entirely of deuterons and have found that including the $\mathrm{D}$ recoil increases $d_{\text {therm }}$ by $40 \%$. A smaller correction can be expected in compounds which include heavier isotopes.

Finally we make the reasonable approximation that neutrons can only be absorbed after they have thermalized. The probability that an interaction after thermalization leads to absorption is

$p_{\mathrm{abs}}=\frac{\sum_{i} \rho_{i} \sigma_{\mathrm{abs}}^{i}}{\sum_{i} \rho_{i}\left(\sigma_{\text {elastic }}^{i}+\sigma_{\mathrm{abs}}^{i}\right)}$.

Therefore one expects that after thermalization a neutron will scatter $1 / p_{\text {abs }}$ times before being absorbed, during which it 
Table 1 Elastic scattering and absorption cross sections of various isotopes

\begin{tabular}{llllllll}
\hline & $\mathrm{H}$ & $\mathrm{D}$ & ${ }^{6} \mathrm{Li}$ & ${ }^{7} \mathrm{Li}$ & $\mathrm{Be}$ & $\mathrm{O}$ & $\mathrm{F}$ \\
\hline$\sigma_{\text {elastic }}$ (barns) & 82.03 & 7.64 & 0.97 & 1.4 & 7.63 & 4.232 & 4.018 \\
$\sigma_{\text {abs }}$ (barns) & 0.3326 & $5.19 \times 10^{-4}$ & 940 & 0.0454 & 0.0076 & $1.9 \times 10^{-4}$ & 0.0096 \\
\hline
\end{tabular}

travels an expected distance of

$d_{\mathrm{abs}}=\sqrt{\frac{2}{3 \pi p_{\mathrm{abs}}} \lambda}$

in a given direction, for example in the radial direction. We will make the rough approximation that $\lambda$ is the same before and after thermalization.

Thermalized neutrons in general will be absorbed in the converter. The probability that a thermalized neutron is absorbed by ${ }^{7} \mathrm{Li}$ and therefore yields a $\bar{v}_{e}$ is

$p_{v}=\frac{\left(\rho_{7} \mathrm{Li}\right)\left(\sigma_{\mathrm{abs}}^{7}\right)}{\sum_{i} \rho_{i} \sigma_{\mathrm{abs}}^{i}}$.

We will be interested in five different converter materials. In each the D will be $99 \%$ isotopically pure (99\% mole fraction), with the remaining $1 \%$ being $\mathrm{H}$. Also the ${ }^{7} \mathrm{Li}$ will be $99.99 \%$ isotopically pure, with the remaining $0.01 \%$ consisting of ${ }^{6} \mathrm{Li}$. In practice the vendors with whom we have spoken offer much lower prices if there are some other impurities, however these other impurities are irrelevant here due to their low neutron absorption cross sections. The five materials are pure metallic $\mathrm{Li}, \mathrm{LiOD}, \mathrm{LiOD} \cdot \mathrm{D}_{2} \mathrm{O}$, a heavy water solution which is $11.6 \%$ LiOD by mass and finally FLiBe. The first material has been chosen in most IsoDAR proposals [45], the last in IsoDAR at KamLAND [47], and the others have been suggested in Ref. [52].

Each compound can have various densities and bulk densities depending on the crystalline structure and/or preparation. We have chosen not to optimize these densities, but rather we have chosen the densities which appear most often on the web pages of vendors, as these are likely to be the most readily available. These densities were then rescaled to the isotope specifications of interest for our study. The results are summarized in Table 2. In the case of the heavy water solution we simply used the density of heavy water. The density of metallic lithium has an appreciable temperature dependence, and we have used a density corresponding to room temperature.

Combining the number densities $\rho_{i}$ in Table 2, together with the cross sections in Table 1 , one can now evaluate the various quantities above for each converter. The results are shown in Table 3. As we have made the approximation that only $\mathrm{D}$ moderates, the thermalization distance for the metallic $\mathrm{Li}$ and the FLiBe converters can not be evaluated. In the other cases, the longest distance is the absorption distance $d_{\text {abs }}$ which is approximately $9 \mathrm{~cm}$ for $\mathrm{LiOD}$ and $\mathrm{LiOD} \cdot \mathrm{D}_{2} \mathrm{O}$ and $22 \mathrm{~cm}$ for the solution.

As can be seen in Eq. (2.6), the absorption distance is determined by two quantities: the mean free path $\lambda$ and the probability of absorption per collision $p_{\text {abs }}{ }^{6} \mathrm{Li}$ is the dominant absorber in each case, so a high concentration of Li leads to a high $p_{\text {abs }}$ and so a low $d_{\mathrm{abs}}$. For example, the solution has a low concentration of $\mathrm{Li}$ and a large $d_{\mathrm{abs}}$. The exception to this rule is metallic $\mathrm{Li}$, whose low $\sigma_{\text {elastic }}$ leads to a large $\lambda$. As a result, neutrons can travel long distances unimpeded in metallic Li, and so it has the longest $d_{\text {abs }}$. Similarly $d_{\text {therm }}$ is in general lowest for the compounds with the highest concentrations of $\mathrm{D}$, which is efficient both for slowing and for scattering neutrons. However LiOD $\cdot \mathrm{D}_{2} \mathrm{O}$ thermalizes neutrons slightly more quickly than the solution due to its higher density.

One expects that the ${ }^{8} \mathrm{Li}$ production, and so the $\bar{\nu}_{e}$ production per neutron will saturate to $p_{v}$ when the converter radius is sufficiently large. The saturation value $p_{v}$ is much less for the solution, but comparable for the other converters.

The fact that $p_{v}$ is close to $1 / 3$ is easy to understand. Apart from the solution, whose high D content implies that $30 \%$ of neutrons are absorbed by $\mathrm{H}$, in all other materials $\mathrm{Li}$ is responsible for at least $85 \%$ of neutron absorption. There is $10^{4}$ times more ${ }^{7} \mathrm{Li}$ than ${ }^{6} \mathrm{Li}$ in each case, but $\sigma_{\text {abs }}$ of ${ }^{6} \mathrm{Li}$ is $2 \times 10^{4}$ times higher than that of ${ }^{7} \mathrm{Li}$. Therefore ${ }^{6} \mathrm{Li}$ absorbs twice as many neutrons as ${ }^{7} \mathrm{Li}$, leaving about $1 / 3$ of the neutrons for ${ }^{7} \mathrm{Li}$. Metallic $\mathrm{Li}$ has the highest value of $p_{v}$ as it contains no other neutron absorbers, whereas the solution has the lowest due to its high $\mathrm{H}$ content. If on the other hand the ${ }^{7} \mathrm{Li}$ purity is increased to $99.995 \%$ as in Ref. [47], then the same argument implies that $p_{v}$ will be about $1 / 2$, corresponding to a $50 \%$ increase in the $\bar{v}_{e}$ yield. We hope that the optimizations described in this note may lead to a smaller, more efficient converter which in turn would allow, at the same price, a higher isotopic purity of Li and so a higher $\bar{v}_{e}$ yield.

\subsection{Simulation results}

The above analytic model uses crude approximations to provide a qualitative understanding of the thermalization and absorption. We will now remove those approximations and report the quantitative results of our neutron transport simulations. 
Table 2 Densities and isotope number densities in various converters. The densities have been rescaled from the original references to reflect the desired isotope compositions

\begin{tabular}{llllll}
\hline & $\mathrm{Li}$ & $\mathrm{LiOD}$ & $\mathrm{LiOD} \cdot \mathrm{D}_{2} \mathrm{O}$ & Solution & FLiBe \\
\hline Density $\left(\mathrm{gm} / \mathrm{cm}^{3}\right)$ & 0.534 & $1.52[54]$ & $1.62[54]$ & 1.1 & $1.94[55]$ \\
$\rho_{\mathrm{H}}\left(\mathrm{cm}^{-3}\right)$ & 0 & $3.66 \times 10^{20}$ & $6.50 \times 10^{20}$ & $6.16 \times 10^{20}$ & 0 \\
$\rho_{\mathrm{D}}\left(\mathrm{cm}^{-3}\right)$ & 0 & $3.62 \times 10^{22}$ & $6.44 \times 10^{22}$ & $6.10 \times 10^{22}$ & 0 \\
$\rho_{6}{ }_{\mathrm{Li}}\left(\mathrm{cm}^{-3}\right)$ & $4.59 \times 10^{18}$ & $3.66 \times 10^{18}$ & $2.17 \times 10^{18}$ & $3.24 \times 10^{17}$ & $2.36 \times 10^{18}$ \\
$\rho_{7} \mathrm{Li}\left(\mathrm{cm}^{-3}\right)$ & $4.59 \times 10^{22}$ & $3.66 \times 10^{22}$ & $2.17 \times 10^{22}$ & $3.24 \times 10^{21}$ & $2.36 \times 10^{22}$ \\
$\rho_{\mathrm{Be}}\left(\mathrm{cm}^{-3}\right)$ & 0 & 0 & 0 & 0 & $1.18 \times 10^{22}$ \\
$\rho_{\mathrm{O}}\left(\mathrm{cm}^{-3}\right)$ & 0 & $3.66 \times 10^{22}$ & $4.34 \times 10^{22}$ & $3.58 \times 10^{22}$ & 0 \\
$\rho_{\mathrm{F}}\left(\mathrm{cm}^{-3}\right)$ & 0 & 0 & 0 & 0 & $4.72 \times 10^{22}$ \\
\hline
\end{tabular}

\begin{tabular}{llllll}
\hline & $\mathrm{Li}$ & $\mathrm{LiOD}$ & $\mathrm{LiOD} \cdot \mathrm{D}_{2} \mathrm{O}$ & Solution & FLiBe \\
\hline$\lambda(\mathrm{cm})$ & 15.5 & 1.95 & 1.32 & 1.52 & 3.20 \\
$p$ & 0 & 0.540 & 0.648 & 0.709 & 0 \\
$d_{\mathrm{D}}(\mathrm{cm})$ & $\infty$ & 1.22 & 0.754 & 0.832 & $\infty$ \\
$d_{\text {therm }}(\mathrm{cm}) E=1$ & $\infty$ & 7.15 & 4.41 & 4.87 & $\infty$ \\
$d_{\text {abs }}(\mathrm{cm})$ & 23.8 & 8.92 & 9.25 & 21.9 & 13.4 \\
$p_{v}$ & 0.326 & 0.317 & 0.300 & 0.208 & 0.280 \\
\hline
\end{tabular}

Table 3 Neutron transport properties of each converter
Second, neutron multiplication increases the efficiency. The latter effect is dominant in FLiBe as ${ }^{9} \mathrm{Be}$ multiplies more efficiently than $\mathrm{D}$ due to the lower energy cost to remove a valence neutron from ${ }^{9} \mathrm{Be}$ with respect to $\mathrm{D}$. Therefore in general FLiBe is better for very high energy neutrons.

We will see below that for the beams considered here, the number of neutrons with energies above $10 \mathrm{MeV}$ is negligible and so neutron multiplication will be inconsequential. However, a high energy deuteron beam will create forward neutrons at half of the deuteron energy. Thus an IsoDAR experiment at a deuteron beam may want to use a FLiBe converter in the forward direction from the target. A $50 \mathrm{MeV}$, $10 \mathrm{~mA}$ deuteron beam is now being built at a user facility in Ningde, China and an upgrade to $200 \mathrm{MeV}$ is foreseen. A hybrid converter, consisting of FLiBe in the forward the direction and a $\mathrm{D}$ rich compound elsewhere, could provide an optimal design for an IsoDAR experiment at this beam.

The cost of the converter is driven by the pure ${ }^{7} \mathrm{Li}$ and so it is reasonable to compare converters at fixed ${ }^{7} \mathrm{Li}$ mass. However, one can obtain the total mass from the ${ }^{7} \mathrm{Li}$ mass by multiplying by $3.57,6.43,30.8,1$ or 7.07 for LiOD, LiOD . $\mathrm{D}_{2} \mathrm{O}$, solution, metallic $\mathrm{Li}$ and FLiBe converters respectively. The radius of the target station as a function of the Li mass is shown in Fig. 3. Such conversions may be of interest if mass and or space are more important constraints than costs, for example for underground configurations.

One may observe that on the right side of each panel in Fig. 1, as the mass is sufficient to thermalize and absorb the neutrons, in general the ${ }^{8} \mathrm{Li}$ production per neutron, which is equal to the $\bar{v}_{e}$ production, reaches an asymptotic value. This 

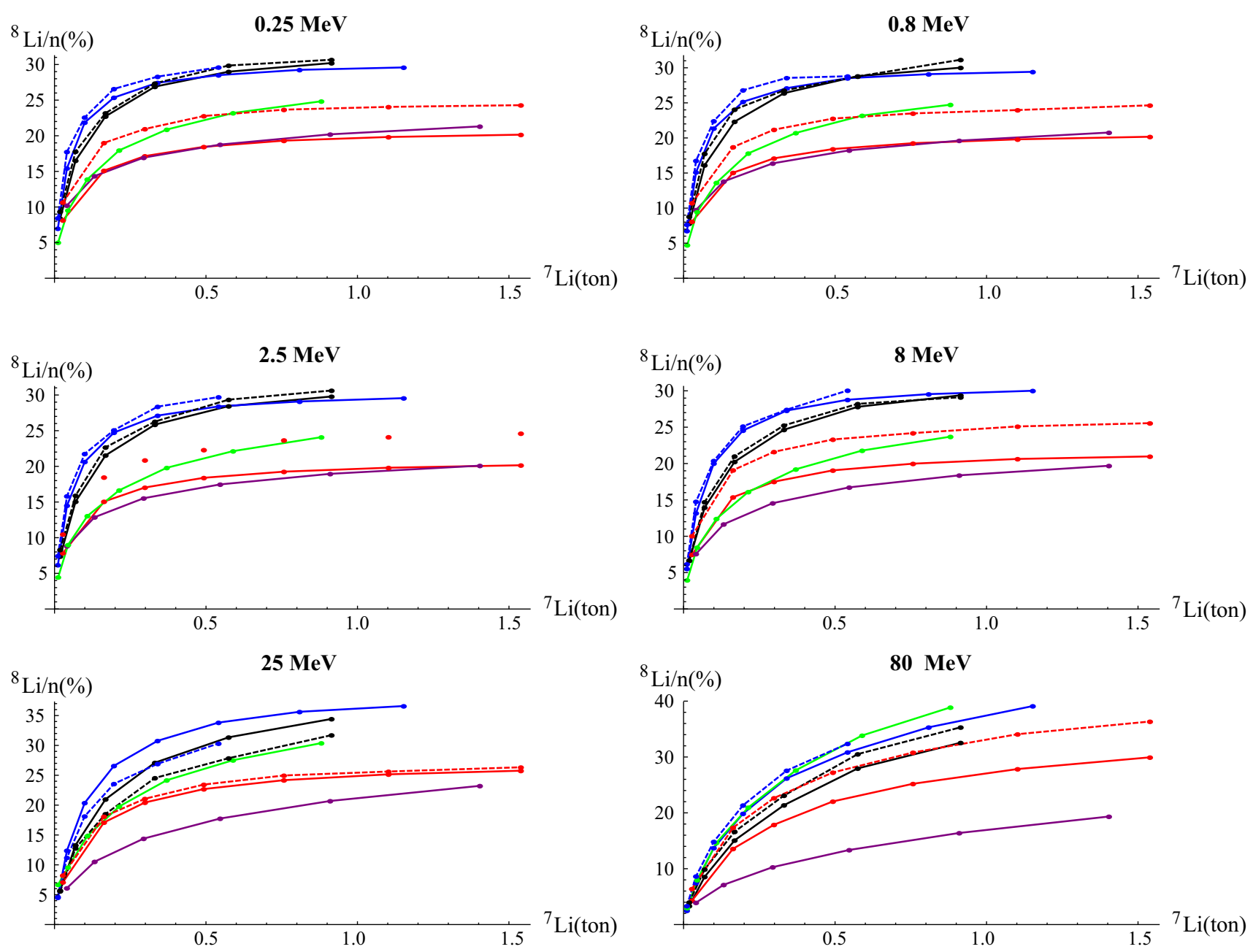

Fig. 1 The ${ }^{8} \mathrm{Li} /$ neutron ratio for monochromatic neutrons at various energies. The black, blue, red, purple and green curves represent the $\mathrm{LiOD}$, LiOD $\cdot \mathrm{D}_{2} \mathrm{O}$, solution, metallic $\mathrm{Li}$ and FLiBe converters respec-

tively. Solid curves were produced with FLUKA and dashed curves with GEANT4. The horizontal axis is the mass of the ${ }^{7} \mathrm{Li}$
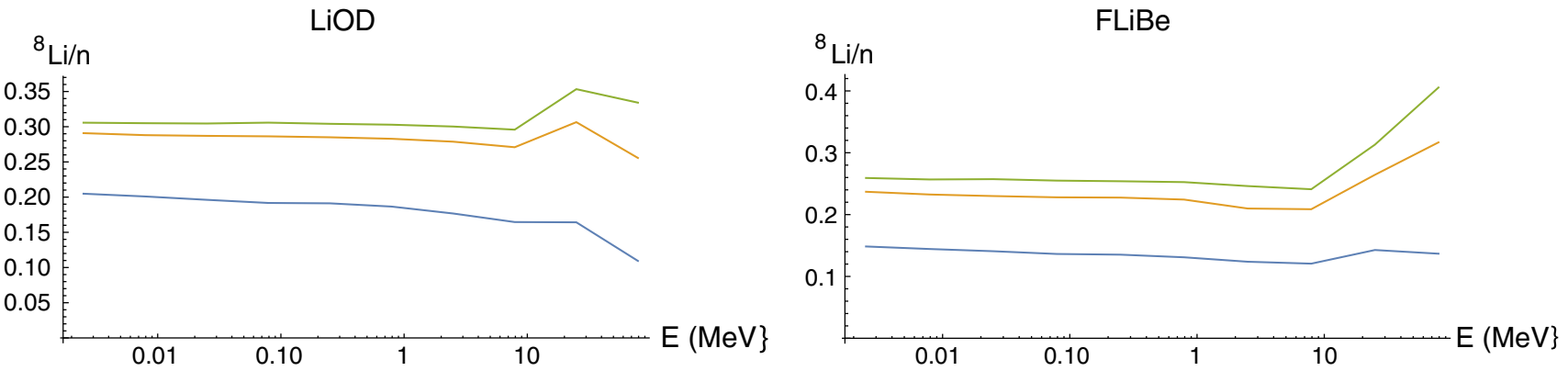

Fig. 2 The ${ }^{8} \mathrm{Li} /$ neutron ratio for monochromatic neutrons at various energies in LiOD (left) and FLiBe (right). The blue, yellow and green curves represent the $0.1,0.5$ and 1 ton of ${ }^{7} \mathrm{Li}$ respectively

asymptotic value agrees well with $p_{v}$ calculated in Eq. (2.7) in every case except for the GEANT4 simulation of the solution, which tends to be about $4 \%$ too high. Note that at energies above $2.5 \mathrm{MeV}$ there is no asymptotic value, instead the ${ }^{8} \mathrm{Li}$ production continues to increase as the converter size is increased. This is because at these energies the neutrons have sufficient energy to break the D or Be in the converter, freeing more neutrons. The neutron increase at these high energies is therefore a result of neutron multiplication. While this neutron multiplication is significant at energies of $25 \mathrm{MeV}$ and 


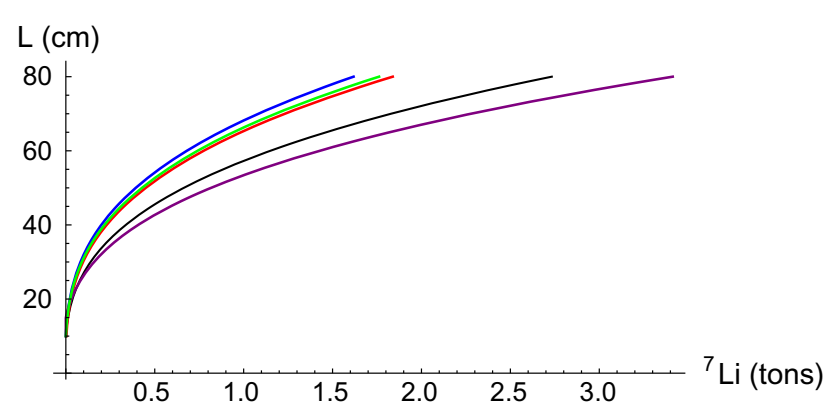

Fig. 3 The target station radius, including the detector and the converter as a function, of the ${ }^{7} \mathrm{Li}$ mass. The black, blue, red, purple and green curves represent the $\mathrm{LiOD}, \mathrm{LiOD} \cdot \mathrm{D}_{2} \mathrm{O}$, solution, metallic $\mathrm{Li}$ and $\mathrm{FLiBe}$ converters respectively. The radius is divided by two in the case of the solution, for clarity of the plot

above, we will see that even $250 \mathrm{MeV}$ protons create very few neutrons above $5 \mathrm{MeV}$, and so neutron multiplication in fact is insignificant in every case that we will consider. In fact, we have run additional simulations with fictional converter materials that have a higher Be density and we have found that at $60 \mathrm{MeV}$ they outperform all of the materials considered here.

One exception to this argument is metallic $\mathrm{Li}$, which does not arrive at an asymptotic value. This is due to the fact that neutrons which are not already thermalized by the heavy water moderator require several meters of $\mathrm{Li}$ to thermalize, and so do not thermalize for the converter sizes that we have considered. For this reason, proposals for IsoDAR experiments using metallic Li converters generally use several tons of Li. Even with such larger converters, the asymptotic ${ }^{8} \mathrm{Li} / \mathrm{n}$ ratio will be less than $p_{v}$ due to absorption of neutrons in the moderator.

The main result of our paper is quite clear in every panel of Fig. 1. The metallic Li converter outside of a heavy water moderator, which has been chosen at many IsoDAR experiments [45], in fact has an appreciably lower neutron yield than the two other solid converters considered when the $\mathrm{Li}$ mass is less than 1.5 tons. This is true for every neutron energy, and so it will be true for every proton beam. The effect is quite large and suggests that by mixing the moderator and the converter one may increase the $\bar{v}$ flux by as much as 50\%. This result has been anticipated in Ref. [52], although quantitatively our simulation results are quite different [59].

\subsection{Thermalization and absorption distances}

To better understand the results of these simulations, in this subsection we will report the results of FLUKA simulations of a simplified geometry designed to determine the thermalization and absorption distances. For this aim, we will consider solid, spherical converters of various radii with no reflector. We will not consider metallic $\mathrm{Li}$, as in IsoDAR pro- posals this is always used in conjunction with a moderator. All neutrons will be created in the centre of the sphere at $1 \mathrm{MeV}$.

A neutron will thermalize or be absorbed inside of the moderator only if the maximal distance in its $3 \mathrm{~d}$ random walk is less than the radius of the sphere. The expected maximal distance in 3 dimensions exceeds the expected final distance in 1 dimension by a factor of $\sqrt{6}$. Therefore one expects, for example, that most neutrons will thermalize if the radius exceeds $\sqrt{6} d_{\text {therm }}$. Similarly, one expects that most neutrons will be absorbed when the radius exceeds

$r=\sqrt{6\left(d_{\mathrm{therm}}^{2}+d_{\mathrm{abs}}^{2}\right)}$.

In practice there are a number of corrections to this idealized estimate. For example, the finite recoil, in particular of D in the target, will increase these distances by up to $40 \%$. Also resonances in the neutron scattering cross section, which generally occur at $100 \mathrm{~s}$ of $\mathrm{keV}$ and exceed the average cross section by an order of magnitude. Our analytical calculation was performed with energy-averaged cross sections, however the resonances provide a considerable contribution to these average cross sections. For example, they contribute nearly one third of the average elastic cross section of neutrons on ${ }^{7} \mathrm{Li}$. As a result, neutrons lose energy quickly until about $100 \mathrm{keV}$, but most of the thermalization distance is traveled by neutrons below these resonances, where the elastic cross section is reduced. This has the effect of increasing the true thermalization distance by several 10s of percent. We have checked that these resonances are correctly implemented in both our FLUKA and GEANT4 simulations, in the former by considering scattering off of a thin target and in the latter by calculating the average trajectory length before a $1 \mathrm{MeV}$ neutron reaches a specific energy.

Our results are summarized in Fig. 4. On the left, we plot the fraction of neutrons which escape from a converter of various radii. FLiBe and the solution are the worst absorbers, as the former is a poor moderator and so has a long thermalization distance while the later is a poor absorber, with $d_{\mathrm{abs}}$ equal to roughly $22 \mathrm{~cm}$. The best absorption is achieved by $\mathrm{LiOD}$ and $\mathrm{LiOD} \cdot \mathrm{D}_{2} \mathrm{O}$, which are adequate moderators and absorbers with $r$ equal to 28 and $25 \mathrm{~cm}$ respectively. One can see that half of the neutrons are absorbed when the radius is about $40 \mathrm{~cm}$. This is somewhat larger than the value of $r$ found in the naive analytic model.

In the right panel we study the thermalization distance by plotting the percentage of neutrons which never reach $0.028 \mathrm{eV}$ before being absorbed or escaping the converter. This is roughly equal to the percentage of neutrons which is not thermalized. FLiBe is by far the worst performer, as it is a poor moderator. On the other hand, most neutrons in $\mathrm{LiOD} \cdot \mathrm{D}_{2} \mathrm{O}$ and the solution thermalize by $30 \mathrm{~cm}$. This is about twice the thermalization radius $\sqrt{6} d_{\text {therm }}$ predicted in 


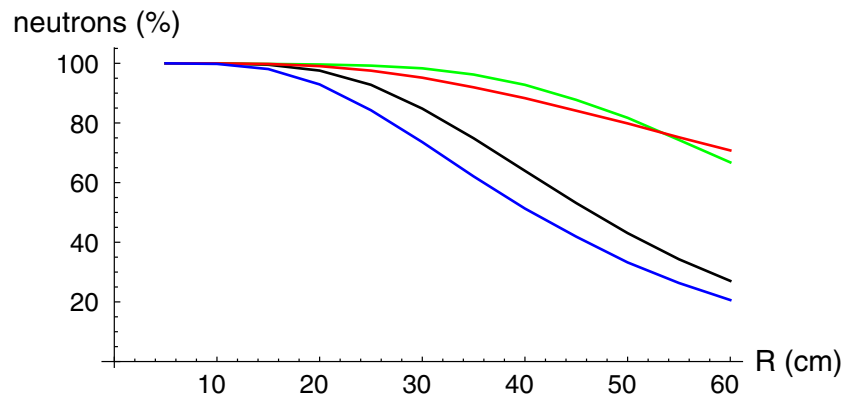

Fig. 4 (Left) The percentage of initially monochromatic $1 \mathrm{MeV}$ neutrons which escape a spherical converter. (Right) The percentage of initially monochromatic $1 \mathrm{MeV}$ neutrons whose energy never falls below

our naive analytic model, in part due to the finite nuclear recoils. Therefore we see that while the analytic model successful predicts the relative performances of the converters, it somewhat underestimates the distances.

In the case of LiOD, the right panel yields a thermalization distance of $37 \mathrm{~cm}$ while the left panel yields a thermalization plus absorption (sum in quadrature) distance of $45 \mathrm{~cm}$. Thus the thermalization distance is greater than the absorption distance, in contrast with the analytic results which do not include the nuclear recoil contribution to the distances. $\mathrm{LiOD} \cdot \mathrm{D}_{2} \mathrm{O}$ is a better moderator and so these distances are 25 and $39 \mathrm{~cm}$. In this case the absorption distance exceeds the thermalization distance. In both cases, the absorption distance yields a nontrivial contribution to the sum in quadrature.

\section{Neutron production}

We will be interested in IsoDAR experiments which begin with a proton beam that strikes a target creating neutrons which are then absorbed by various isotopes. Those absorbed by ${ }^{7} \mathrm{Li}$ provide ${ }^{8} \mathrm{Li}$ and so our $\bar{v}_{e}$ signal. In Sect. 2 we studied the second step of this experiment, the neutron transport and absorption. In this section we will instead describe the first step, the production of neutrons at the target.

In each case, throughout this paper, the target will be $20 \mathrm{~cm}$ long with a $10 \mathrm{~cm}$ radius. We have optimized the target dimensions in each case so as to maximize ${ }^{8} \mathrm{Li} / \mathrm{p}$. We have found that this standard size yields a ${ }^{8} \mathrm{Li}$ production rate which is near the optimum value for the three proton beam energies considered, and so for simplicity we report only simulations with this fixed size. The 25 and $60 \mathrm{MeV}$ proton beams always strike a Be target, whereas we consider heavy metal targets for the $250 \mathrm{MeV}$ beam, as these produce a higher neutron yield above about $50 \mathrm{MeV}$.

We have simulated this production with GEANT4 and FLUKA and we have compared our results with experimen-

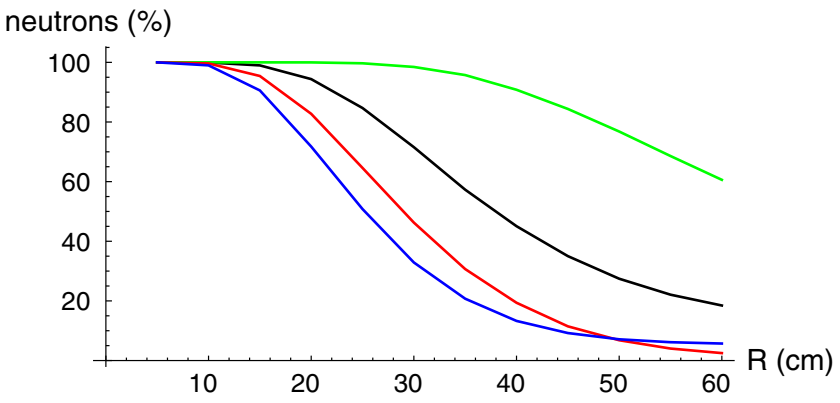

$0.028 \mathrm{eV}$ before escaping or being absorbed. The black, blue, red and green curves represent the $\mathrm{LiOD}, \mathrm{LiOD} \cdot \mathrm{D}_{2} \mathrm{O}$, solution and FLiBe converters respectively. The horizontal axis is the radius of the converter

tal data at various energies up to $100 \mathrm{MeV}[60,61]$ and also with the simulations of Ref. [62] at $250 \mathrm{MeV}$. In general we have found that the GEANT4 simulations yield 10-20\% less neutrons than experimental data and the FLUKA simulations $20-40 \%$ less, whereas we found better than $1 \%$ agreement with the simulations of Ref. [62]. The deficit in neutron production in FLUKA arises entirely at low energies.

Including the latest data it is possible to improve the GEANT4 simulations considerably [47]. However one of the main results of Sect. 2 is that below about $25 \mathrm{MeV}$ the initial neutron energy has little effect on the isotope production. In Fig. 5 we plot the normalized cumulative distributions of the neutron spectra produced by 25,60 and $250 \mathrm{MeV}$ proton beams, as determined by FLUKA.

As can be seen in Fig. 5, in each case less than 5\% of the neutrons exiting the target have energies in excess of $25 \mathrm{MeV}$ and at most about $10 \%$ have energies in excess of $10 \mathrm{MeV}$. We have seen in Sect. 2.2 that below $10 \mathrm{MeV}$, the ${ }^{8} \mathrm{Li}$ yield per neutron is quite independent of the neutron energy. Therefore the shape of the neutron spectrum will have very little effect on the final ${ }^{8} \mathrm{Li}$ yield, especially for a large target station. This means that for our study of the effect of target station design on the ${ }^{8} \mathrm{Li}$ yield, we only need the total normalizations of the neutron flux and not the detailed spectral shape below $10 \mathrm{MeV}$. For example, even if the neutron energy is doubled in a $100 \mathrm{~kg} \mathrm{LiOD}$ converter, the ${ }^{8} \mathrm{Li}$ yield only falls about $5 \%$. This justifies our use of unmodified GEANT4 and FLUKA in this note: FLUKA and GEANT4 underestimate the neutron flux significantly but the missing neutrons are at energies well below the neutron multiplication threshold, where the neutron energy and so the spectral shape does not affect the ${ }^{8} \mathrm{Li}$ yield. It would therefore be possible to correct for this shortfall of neutrons by rescaling the ${ }^{8} \mathrm{Li}$ yield by the ratio of neutrons observed in a fixed target experiment such as $[60,61]$ to those obtained by the simulation. In Fig. 5, we plot the fractional distribution of neutrons and so the overall normalisation does not appear. In the following section our results are not rescaled. 


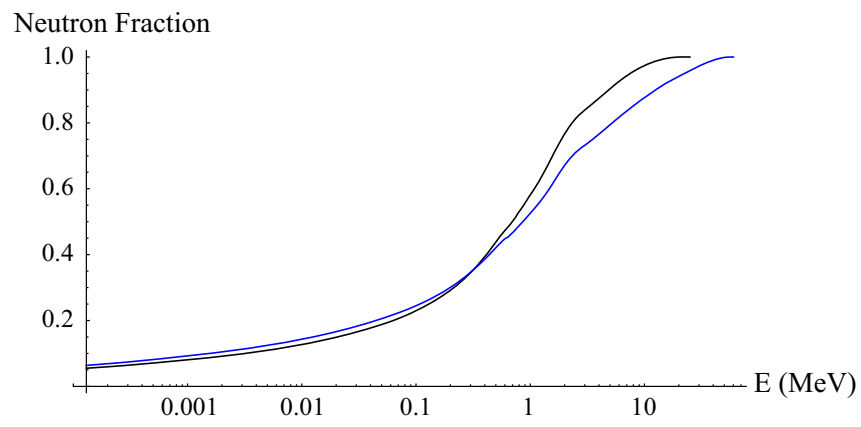

Fig. 5 The normalized cumulative distribution of the neutron energies upon exiting the target as produced using a $25 \mathrm{MeV}$ (left panel, black), $60 \mathrm{MeV}$ (left panel, blue) and $250 \mathrm{MeV}$ (right panel) proton beam. The

\section{The full simulation}

In this subsection we simulate the full experimental setup, from the proton beam to the ${ }^{8} \mathrm{Li}$ production. Note that the result cannot simply be obtained by folding the results of Sect. 3 into Sect. 2 because neutrons can bounce from the converter back into the target, where they may be absorbed. This bounce-back process is only possible in a simulation which includes both the target and the converter. In particular, we will see that bounce-back is most important for $\mathrm{W}$ targets, which have the highest probability of absorbing the neutrons. On the other hand it is nearly negligible for the other targets. The $\mathrm{W}$ target nonetheless is important as a granular $\mathrm{W}$ target is currently the favored target for the CI-ADS $250 \mathrm{MeV}$ beam, even if the beam energy is increased to $600 \mathrm{MeV}$.

As FLUKA predicts lower spallation neutron yields than have been observed in experiment, one may expect that the true ${ }^{8} \mathrm{Li}$ yields will be $20-40 \%$ greater than those reported below in each case.

\subsection{Comparison of converters}

In this subsection we compare various converter designs. As bounce-back results in significant neutron loss in the case of a W converter, the target has been surrounded with a $10 \mathrm{~cm}$ gap or vacuum sleeve in this case as described in Sect. 4.3. To increase the yield of the metallic Li converter, $5 \mathrm{~cm}$ of heavy water moderator has been placed between the target and the converter in this case, following the design in Ref. [45].

The results are shown in Fig. 6 for various ${ }^{7} \mathrm{Li}$ masses. Our main result is apparent here, the converters in which $\mathrm{Li}$ is mixed with a deuterium moderator significantly outperform the others with the same total mass of ${ }^{7} \mathrm{Li}$, in accordance with the expectations of Ref. [52]. The ${ }^{7} \mathrm{Li}$ mass dominates the materials cost of the converter, however in Fig. 7 we have performed the same comparison fixing the total converter mass. Here one finds that metallic $\mathrm{Li}$ is the best at very small

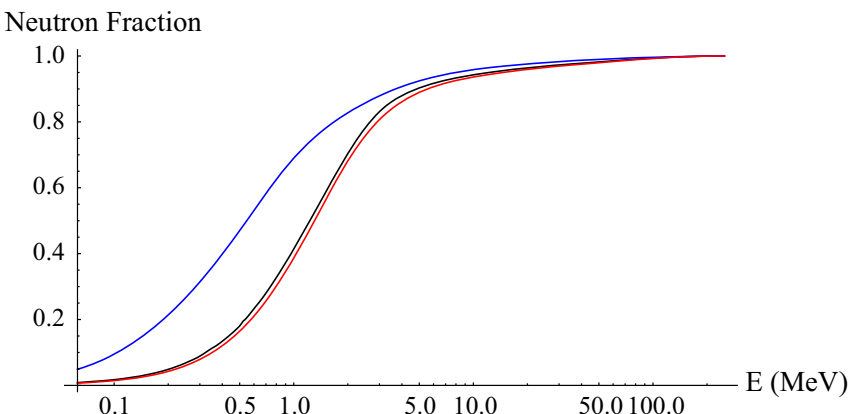

$y$-axis is the fraction of neutrons beneath a specific energy. On the left panel a Be target is used, while on the right panel $\mathrm{Pb}$ (black), W (blue) and $\mathrm{Bi}$ (red) targets are used

masses. In the case of the $\mathrm{W}$ target and $250 \mathrm{MeV}$ beam, LiOD $\cdot \mathrm{D}_{2} \mathrm{O}$ suffers considerably from neutrons lost after bouncing back into the target and indeed one can see that as a result, at fixed total converter mass, it is outperformed by metallic Li.

\subsection{Liquid nitrogen cooling}

As can be seen in Table 3, an important contribution to the distance that neutrons need to travel is $d_{\mathrm{abs}}$, the distance traveled between thermalization and absorption. The ${ }^{8} \mathrm{Li}$ to neutron ratio approaches $p_{v}$ when the size of the converter is several times $d_{\mathrm{abs}}$. Therefore $d_{\mathrm{abs}}$ sets the scale of the converter and is responsible for the reduction in ${ }^{8} \mathrm{Li}$ generation when the converter is smaller than that scale. As a result, a smaller $d_{\mathrm{abs}}$ would allow for a smaller converter with the same $p_{v}$ or a larger $p_{v}$ with the same size converter.

The length scale $d_{\mathrm{abs}}$ is, according to Eqs. (2.5) and (2.6), inversely proportional to the square root of the absorption cross sections $\sigma_{\text {abs }}^{i}$. These in turn are inversely proportional to the neutron velocity, and so to the square root of the neutron temperature. This means that if the converter temperature is reduced by a factor of 4 , by cooling with liquid nitrogen, then the neutron velocities will be reduced by a factor of 2 and so $d_{\mathrm{abs}}$ will be reduced by a factor of $\sqrt{2}$, allowing for an increased ${ }^{8} \mathrm{Li}$ yield with a smaller and cheaper target station.

In liquid nitrogen one expects the absorption distance to be halved. On the other hand, the factor of four reduction in temperature corresponds to only 4 or 5 additional D collisions, and so only about a 5\% increase in the thermalization distance. Thus one expects the sum in quadrature of the thermalization and absorption distances to decrease if the converter is cooled to liquid nitrogen temperatures.

We have rerun our simulations with the converter at liquid nitrogen temperature, again without including any cooling system in our design. In practice the 60 and $250 \mathrm{MeV}$ experiments are likely to have enough money to buy large convert- 

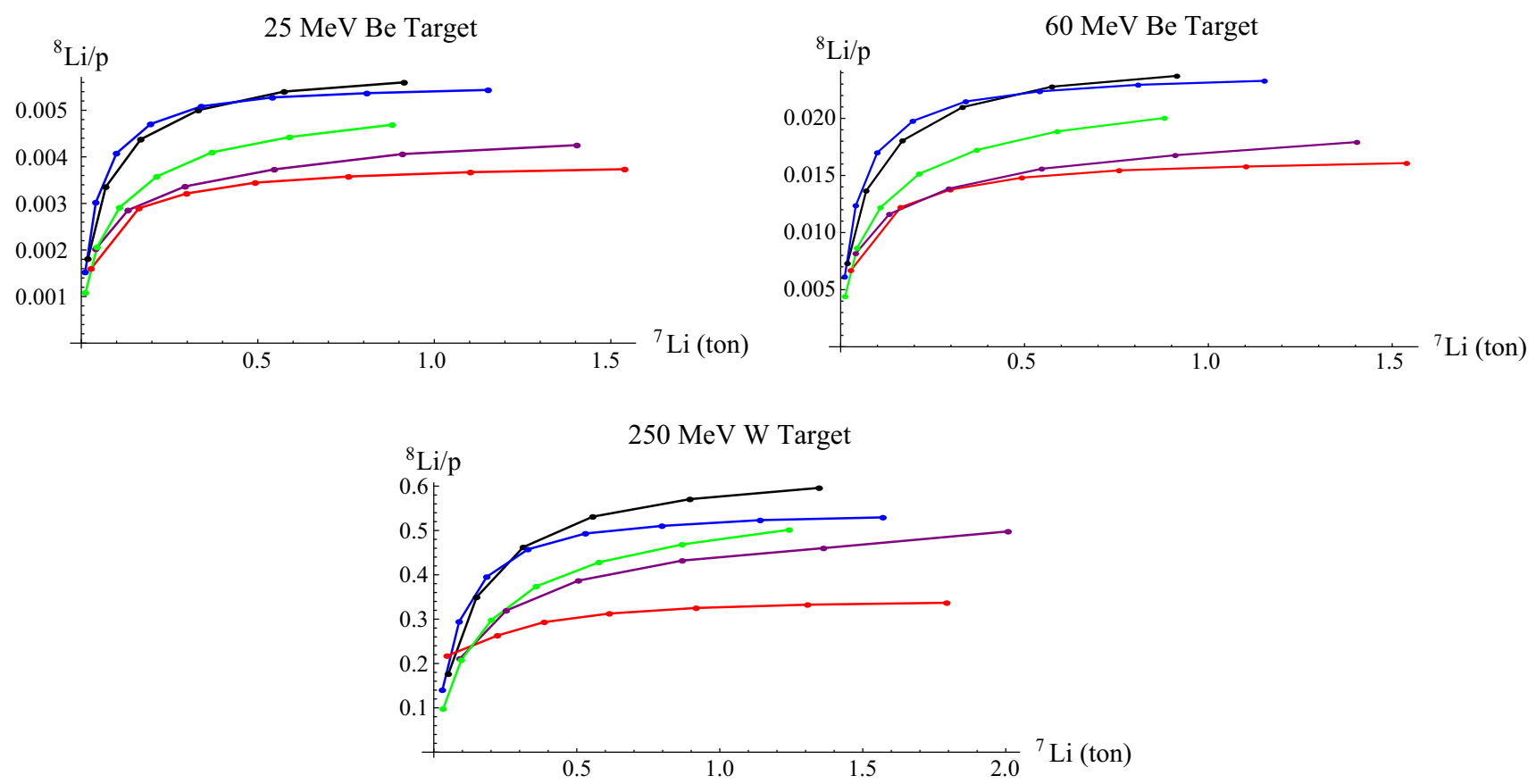

Fig. 6 The ${ }^{8} \mathrm{Li}$ production given a 25,60 and $250 \mathrm{MeV}$ proton beam is shown in the three panels as a function of the ${ }^{7} \mathrm{Li}$ mass. The target is always a cylinder of length $20 \mathrm{~cm}$ and radius $10 \mathrm{~cm}$. At 25 and $60 \mathrm{MeV}$ the target is Be. At $250 \mathrm{MeV}$ the target is $\mathrm{W}$ and it is surrounded by a
$10 \mathrm{~cm}$ vacuum sleeve. The converters are $\mathrm{LiOD}$ (black), $\mathrm{LiOD} \cdot \mathrm{D}_{2} \mathrm{O}$ (blue), the solution (red), metallic Li (purple) and FLiBe (green). In the case of metallic $\mathrm{Li}$, a $5 \mathrm{~cm}$ heavy water moderator is placed inside the converter
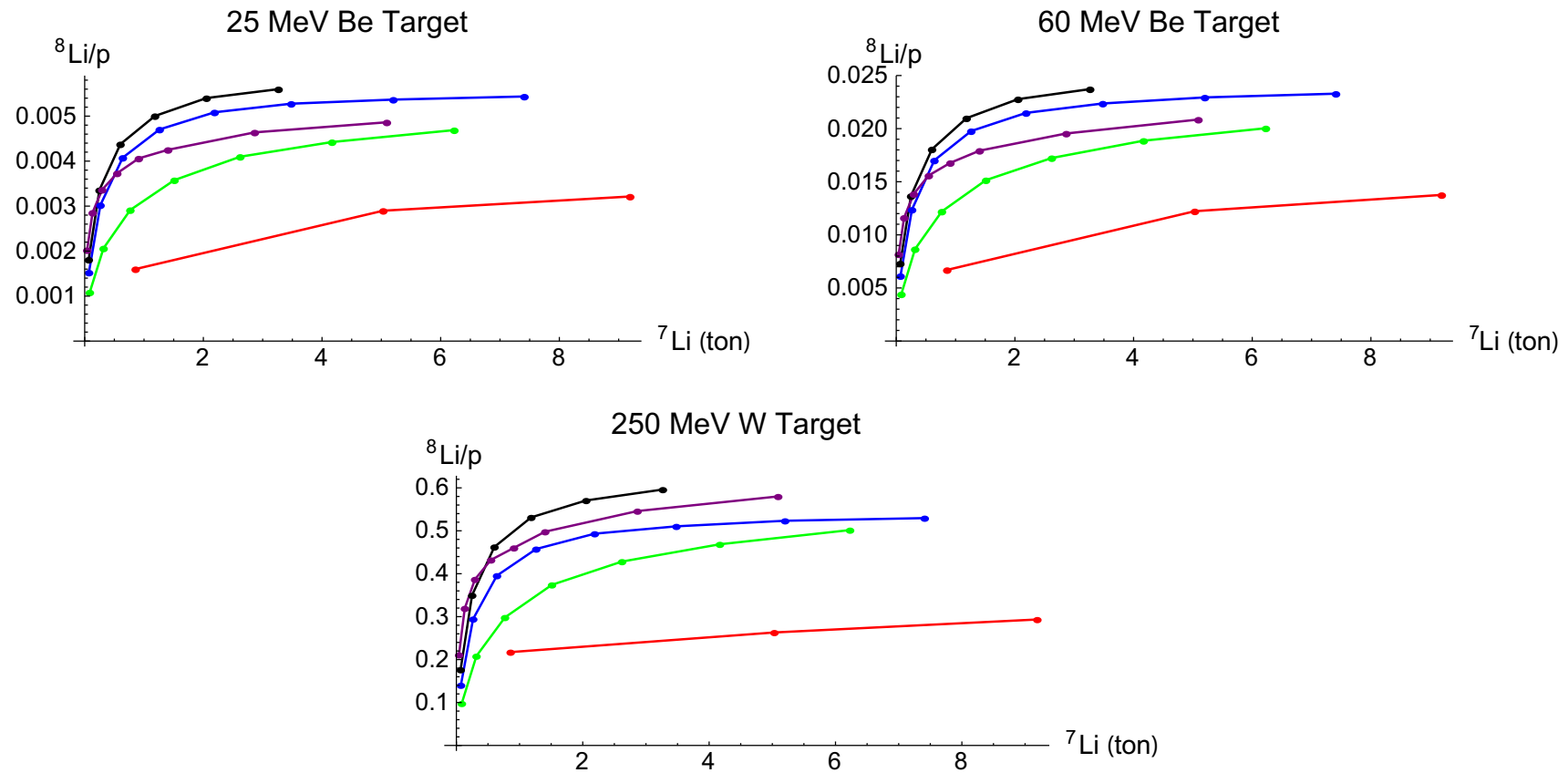

Fig. 7 As in Fig. 6 but the $x$-axis is the total converter mass instead of just the ${ }^{7}$ Li mass

ers, and so for brevity we only report our results in the case of the $25 \mathrm{MeV}$ proton beam in Fig. 8, although the cooling has a similar effect at other energies. As expected based on the general arguments above, liquid nitrogen cooling has only a modest effect on the ${ }^{8} \mathrm{Li}$ production. However, in general it leads to a ${ }^{8} \mathrm{Li}$ production with a mass $M$ of ${ }^{7} \mathrm{Li}$ equal to that which would be obtained with a mass of about $2 M$ of room temperature ${ }^{7} \mathrm{Li}$. This corresponds to an improvement which 


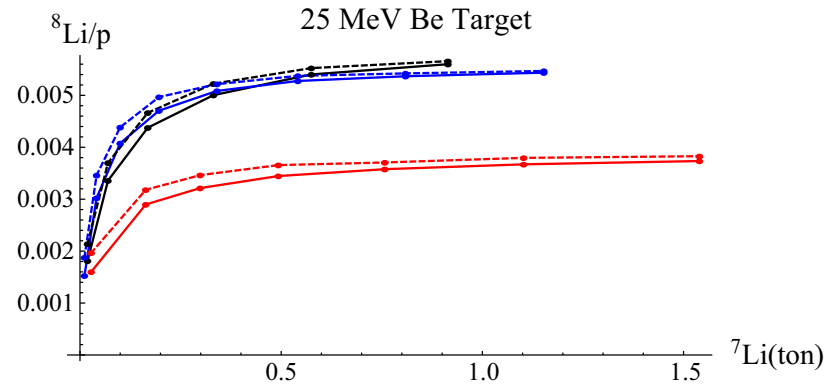

Fig. 8 The ${ }^{8} \mathrm{Li}$ yield per $25 \mathrm{MeV}$ proton as a function of the ${ }^{7} \mathrm{Li}$ mass. The black, blue and red curves correspond to LiOD, LiOD $\cdot \mathrm{D}_{2} \mathrm{O}$ and the solution respectively. The solid (dashed) curves correspond to a room temperature (liquid nitrogen cooled) converter

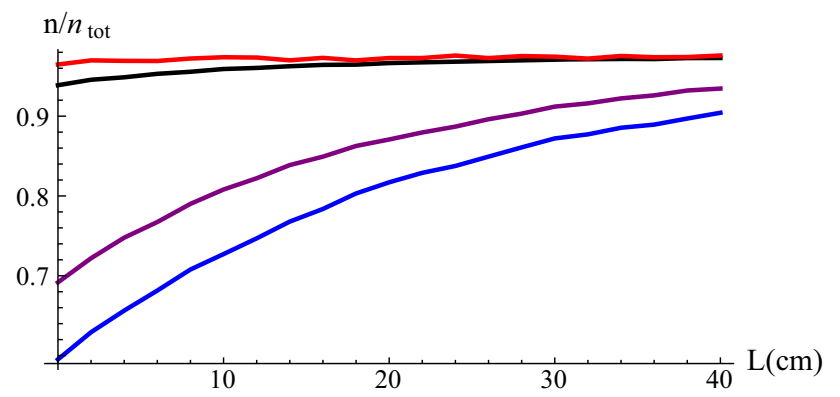

Fig. 9 The fraction of neutrons that are not lost to neutron bounceback, in the case of a $250 \mathrm{MeV}$ proton beam, as a function of the size of the gap between the target and the converter. The red, black, purple and blue curves correspond to a $\mathrm{Bi}$ target with a $\mathrm{LiOD} \cdot \mathrm{D}_{2} \mathrm{O}$ converter, $\mathrm{a} \mathrm{Pb}$ target with a LiOD $\cdot \mathrm{D}_{2} \mathrm{O}$ converter, a W target with a LiOD converter and a $\mathrm{W}$ target with a $\mathrm{LiOD} \cdot \mathrm{D}_{2} \mathrm{O}$ converter

is quite small for large ${ }^{7} \mathrm{Li}$ converter masses, but approaches $20 \%$ when the ${ }^{7} \mathrm{Li}$ mass is less than $100 \mathrm{~kg}$.

\subsection{Vacuum sleeve}

In every setup, a considerable fraction of the neutrons bounce back into the target. However only the $\mathrm{W}$ target has a sufficiently high neutron absorption cross section to absorb an appreciable fraction of these neutrons. Nonetheless, a W target is currently favored for the CI-ADS $250 \mathrm{MeV}$ accelerator, and so this case cannot be ignored.

If there is a gap or vacuum sleeve between the target and the converter, then some of the neutrons bouncing back from the converter will fly through the gap and reenter the converter elsewhere without ever entering the target. As a result, a gap reduces the number of neutrons lost to bounce-back. In Fig. 9 we plot the results of FLUKA simulations of the fraction of neutrons which leave the target and are not reabsorbed in the target later, as a function of the gap size.

One can observe that in the case of the LiOD $\cdot \mathrm{D}_{2} \mathrm{O}$ converter, more neutrons are lost to bounce-back than in the case of the LiOD converter. We have verified that this is a con- sequence of more neutrons bouncing back, and not of the energy spectrum of the bounced-back neutrons.

The main result of this study is that bounce-back can lead to a loss of as many as $40 \%$ of the neutrons. However, with a sufficiently large gap, this loss can be made as small as desired. Of course, a large gap also implies that a greater quantity of ${ }^{7} \mathrm{Li}$ is needed for the same converter thickness. It also means that the $\bar{v}_{e}$ are created over a larger physical area, leading to a greater baseline uncertainty which reduces the sensitivity of a sterile neutrino experiment at large $\Delta \mathrm{M}^{2}$.

\section{Conclusions}

IsoDAR experiments provide powerful and we believe also feasible tests of sterile neutrino models that have been invoked to explain observed anomalies. Several such experiments have been proposed at laboratories around the world.

In this note we have simulated three proposed methods for increasing the $\bar{v}_{e}$ yield of these experiments. In the first, the ${ }^{7} \mathrm{Li}$ converter is mixed with a D moderator. In the second, the converter is cooled to liquid nitrogen temperatures. In the third, a gap is placed between the target and the converter. We have not investigated the feasibility of these modifications. In particular, our simulations were quite idealized as we did not include a support structure for a target separated by a gap, nor cooling for the target or for the converter. We also did not include the impurities such as $\mathrm{K}$ which are normally included in isotopically pure ${ }^{7} \mathrm{Li}$ available on the market.

We have found that the utility of each of these modifications depends on the experimental setup. For example, the purpose of the gap is to allow bounced-back neutrons to reenter the converter without passing through the target where they may be absorbed. However, only the $\mathrm{W}$ target has a sufficiently high absorption cross section for bounced-back neutrons to significantly affect the $\bar{v}_{e}$ yield. Therefore we have found that the gap is only useful in the case of the $\mathrm{W}$ target. However it is quite likely that the $250 \mathrm{MeV}$ CI-ADS beam will use a $\mathrm{W}$ target, therefore it seems likely that one will wish to incorporate this gap in the target station design for any IsoDAR experiment at that beam.

The liquid nitrogen cooling reduces the fraction of neutrons that escape the converter and the reflector to the outside. The fraction of escapes which are prevented is significant. However such neutron escapes are themselves only significant in the case of a thin moderator, in particular if less than $100 \mathrm{~kg}$ of $\mathrm{Li}$ is used. Such a thin moderator would only be used either to save money, or so as to be able to obtain a higher purity at the same price. For a very thin moderator, the increase in $\bar{v}_{e}$ yield at liquid nitrogen temperatures approaches $20 \%$. However, given the hot target in the center of the target station, appreciable cooling of the converter may be impractical. In fact, it may be that the converter is appre- 
ciably above room temperature, in which case more neutrons will escape than we have simulated and so the converter will need to be larger. We need to insure however that the converter does not become too hot, as some of these converters will thermally decompose [63]. In the future we intend to perform more detailed simulations, including heat dissipation, to resolve this issue.

We have found that FLiBe provides the highest ${ }^{8} \mathrm{Li}$ and so $\bar{v}_{e}$ yield for neutron energies well above $25 \mathrm{MeV}$. However we have also found that proton beams of energy up to $250 \mathrm{MeV}$ produce negligible quantities of neutrons at such high energies. As a result, the highest ${ }^{8} \mathrm{Li} / \mathrm{p}$ yields were obtained using Li compounds which include D.

Perhaps our main result is that we have confirmed the claims of Ref. [52] that mixing the converter with a D moderator improves the neutron capture rate appreciably. As a result, for $\mathrm{Li}$ masses of order a ton or less, neutrons can thermalize anywhere in the converter volume and so far less neutrons escape, increasing the $\bar{v}_{e}$ yield considerably with respect to the metallic Li converter outside of a thin moderator proposed in Ref. [45].

In this article we have determined how several modifications of the core IsoDAR target station design can potentially affect the $\bar{v}_{e}$ yield. In the future, to drive these proposals further, we will investigate both their practicality and also their effects on the physics goals of IsoDAR experiments. To do this, we will require simulations which correctly reproduce the shape of the neutron energy spectrum and its angular distribution and also model the target station heating and cooling.

Acknowledgements We thank M. Osipenko for discussions and advice. JE and EC are supported by NSFC Grant 11375201. EC is also supported by the Chinese Academy of Sciences President's International Fellowship Initiative Grant 2015PM063 and NSFC Grant 11605247. MD is supported by the Chinese Academy of Sciences President's International Fellowship Initiative Grant 2016PM043. JE is supported by the CAS Key Research Program of Frontier Sciences Grant QYZDY-SSW-SLH006. JE, EC and MD thank the Recruitment Program of High-end Foreign Experts for support.

Open Access This article is distributed under the terms of the Creative Commons Attribution 4.0 International License (http://creativecomm ons.org/licenses/by/4.0/), which permits unrestricted use, distribution, and reproduction in any medium, provided you give appropriate credit to the original author(s) and the source, provide a link to the Creative Commons license, and indicate if changes were made.

Funded by SCOAP ${ }^{3}$.

\section{References}

1. C. Athanassopoulos et al. (LSND Collaboration), Measurements of the reactions C-12 (electron-neutrino, e-) N-12 (g.s.) and C12 (electron-neutrino, e-) N*-12. Phys. Rev. C 55, 2078 (1997). arXiv:nucl-ex/9705001
2. A. Aguilar-Arevalo et al. (LSND Collaboration), Evidence for neutrino oscillations from the observation of anti-neutrino(electron) appearance in a anti-neutrino(muon) beam. Phys. Rev. D 64, 112007 (2001). arXiv:hep-ex/0104049

3. B. Armbruster et al. (KARMEN Collaboration), Upper limits for neutrino oscillations muon-anti-neutrino $\longrightarrow$ electron-antineutrino from muon decay at rest. Phys. Rev. D 65, 112001 (2002). arXiv:hep-ex/0203021

4. M. Antonello et al. (ICARUS Collaboration), Search for anomalies in the $v_{e}$ appearance from a $v_{\mu}$ beam. Eur. Phys. J. C 73, 2599 (2013). arXiv:1307.4699 [hep-ex]

5. A.A. Aguilar-Arevalo et al. (MiniBooNE Collaboration), Improved Search for $\bar{v}_{\mu} \rightarrow \bar{v}_{e}$ oscillations in the MiniBooNE experiment. Phys. Rev. Lett. 110, 161801 (2013). arXiv:1207.4809 [hep-ex]. arXiv: 1303.2588 [hep-ex]

6. W. Hampel et al. (GALLEX Collaboration), Final results of the Cr51 neutrino source experiments in GALLEX. Phys. Lett. B 420, 114 (1998)

7. J.N. Abdurashitov et al. (SAGE Collaboration), Measurement of the solar neutrino capture rate with gallium metal. III: Results for the 2002-2007 data-taking period. Phys. Rev. C 80, 015807 (2009). arXiv:0901.2200 [nucl-ex]

8. T.A. Mueller et al., Improved predictions of reactor antineutrino spectra. Phys. Rev. C 83, 054615 (2011). arXiv:1101.2663 [hepex]

9. P. Huber, On the determination of anti-neutrino spectra from nuclear reactors. Phys. Rev. C 84, 024617 (2011). arXiv:1106.0687 [hep-ph] [Erratum: Phys. Rev. C 85, 029901 (2012)]

10. G. Mention, M. Fechner, T. Lasserre, T.A. Mueller, D. Lhuillier, M. Cribier, A. Letourneau, The reactor antineutrino anomaly. Phys. Rev. D 83, 073006 (2011). arXiv:1101.2755 [hep-ex]

11. S.B. Kim, Observation of reactor antineutrino disappearance at RENO. Talk given on June 4, 2012 at Neutrino 2012 in Kyoto

12. S.H. Seo (RENO Collaboration), New results from RENO and the $5 \mathrm{MeV}$ excess. AIP Conf. Proc. 1666, 080002 (2015). arXiv: 1410.7987 [hep-ex]

13. Y. Abe et al. (Double Chooz Collaboration), Improved measurements of the neutrino mixing angle $\theta_{13}$ with the Double Chooz detector. JHEP 1410, 086 (2014). arXiv:1406.7763 [hep-ex] [JHEP 1502, 074 (2015)]

14. D.V. Naumov (Daya Bay Collaboration), Recent results from Daya Bay experiment. EPJ Web Conf. 95, 04043 (2015). arXiv: 1412.7806 [hep-ex]

15. A.A. Zakari-Issoufou et al. (IGISOL Collaboration), Total absorption spectroscopy study of ${ }^{92} \mathrm{Rb}$ decay: a major contributor to reactor antineutrino spectrum shape. Phys. Rev. Lett. 115(10), 102503 (2015). arXiv:1504.05812 [nucl-ex]

16. D.A. Dwyer, T.J. Langford, Spectral structure of electron antineutrinos from nuclear reactors. Phys. Rev. Lett. 114(1), 012502 (2015). arXiv:1407.1281 [nucl-ex]

17. F.P. An et al., (Daya Bay Collaboration), Evolution of the reactor antineutrino flux and spectrum at Daya Bay. Phys. Rev. Lett. 118(25), 251801 (2017)

18. P.A.R. Ade et al., (Planck Collaboration), Planck 2015 results. XIII. Cosmological parameters. Astron. Astrophys. 594, A13 (2016). arXiv: 1502.01589 [astro-ph.CO]

19. T. Delubac et al. (BOSS Collaboration), Baryon acoustic oscillations in the Ly forest of BOSS DR11 quasars. Astron. Astrophys. 574, A59 (2015). arXiv:1404.1801 [astro-ph.CO]

20. A.G. Riess et al., A $2.4 \%$ determination of the local value of the Hubble constant. Astrophys. J. 826(1), 56 (2016). arXiv:1604.01424 [astro-ph.CO]

21. J. Evslin, Model-independent dark energy equation of state from unanchored baryon acoustic oscillations. Phys. Dark Univ. 13, 126 (2016). arXiv:1510.05630 [astro-ph.CO] 
22. J. Evslin, Isolating the Lyman alpha forest BAO anomaly. JCAP 04, 024 (2017). arXiv:1604.02809 [astro-ph.CO]

23. E. Ciuffoli, J. Evslin, H. Li, The reactor anomaly after Daya Bay and RENO. JHEP 1212, 110 (2012). arXiv:1205.5499 [hep-ph]

24. Y. Zhang, H. Zhang, D. Wang, Y. Qi, Y. Wang, G.B. Zhao, Probing dynamics of dark energy with latest observations. Res. Astron. Astrophys. 17(6), 050 (2017). arXiv:1703.08293 [astro-ph.CO]

25. E. Di Valentino, A. Melchiorri, E.V. Linder, J. Silk, Constraining dark energy dynamics in extended parameter space. Phys. Rev. D 96(2), 023523 (2017). arXiv:1704.00762 [astro-ph.CO]

26. Talk by N. Bowden, Review of short-baseline reactor antineutrino experiments, July 5, 2016 at Neutrino 2016 in London. Talk available at http://neutrino2016.iopconfs.org/programme (2016)

27. Y.J. Ko et al., Sterile neutrino search at NEOS experiment. Phys. Rev. Lett. 118(12), 121802 (2017). arXiv:1610.05134 [hep-ex]

28. G. Boireau et al. (NUCIFER Collaboration), Online monitoring of the Osiris reactor with the Nucifer neutrino detector. Phys. Rev. D 93(11), 112006 (2016). https://doi.org/10.1103/PhysRevD.93. 112006. arXiv:1509.05610 [physics.ins-det]

29. V. Helaine, Sterile neutrino search at the ILL nuclear reactor: the STEREO experiment. Talk presented at Prospects in Neutrino Physics (NuPhys2015) 16-18 Dec 2015. London, UK. arXiv:1604.08877 [physics.ins-det]

30. I. Alekseev et al., Detector of the reactor AntiNeutrino based on solid-state plastic Scintillator (DANSS). Status and first results. J. Phys. Conf. Ser. 798(1), 012152 (2017)

31. I. Michiels, SoLid: search for oscillation with a ${ }^{6} \mathrm{Li}$ detector at the BR2 research reactor. Poster presented at Prospects in Neutrino Physics (NuPhys2015) 16-18 Dec 2015. London, UK. arXiv:1605.00215 [physics.ins-det]

32. J. Ashenfelter et al. (PROSPECT Collaboration), The PROSPECT physics program. J. Phys. G 43(11), 113001 (2016). arXiv:1512.02202 [physics.ins-det]

33. S. Gariazzo, C. Giunti, M. Laveder, Y.F. Li, E.M. Zavanin, Light sterile neutrinos. J. Phys. G 43, 033001 (2016). arXiv:1507.08204 [hep-ph]

34. S. Gariazzo, C. Giunti, M. Laveder, Y.F. Li, Updated global 3+1 analysis of short-baseline neutrino oscillations. JHEP 1706, 135 (2017). arXiv:1703.00860 [hep-ph]

35. C. Kraus, A. Singer, K. Valerius, C. Weinheimer, Limit on sterile neutrino contribution from the Mainz neutrino mass experiment. Eur. Phys. J. C 73(2), 2323 (2013). arXiv:1210.4194 [hep-ex]

36. A.I. Belesev et al., The search for an additional neutrino mass eigenstate in the $2-100 \mathrm{eV}$ region from 'Troitsk nu-mass' data: a detailed analysis. J. Phys. G 41, 015001 (2014). arXiv:1307.5687 [hep-ex]

37. P. Adamson et al. (MINOS Collaboration), Search for sterile neutrinos mixing with muon neutrinos in MINOS. Phys. Rev. Lett. 117(15), 151803 (2016). arXiv:1607.01176 [hep-ex]

38. M.G. Aartsen et al. (IceCube Collaboration), Searches for sterile neutrinos with the IceCube detector. Phys. Rev. Lett. 117(7), 071801 (2016). arXiv:1605.01990 [hep-ex]

39. J. Alonso, F.T. Avignone, W.A. Barletta, R. Barlow, H.T. Baumgartner, A. Bernstein, E. Blucher, L. Bugel et al., Expression of interest for a novel search for $\mathrm{CP}$ violation in the neutrino sector: DAE $\delta$ ALUS. arXiv:1006.0260 [physics.ins-det] (unpublished)

40. E. Ciuffoli, J. Evslin, F. Zhao, Neutrino physics with accelerator driven subcritical reactors. JHEP 1601, 004 (2016). arXiv:1509.03494 [hep-ph]

41. V.I. Lyashuk, Y.S. Lutostansky, Intense antineutrino source based on a lithium converter. Proposal for a promising experiment for studying oscillations. JETP Lett. 103(5), 293 (2016) [Pisma Zh. Eksp. Teor. Fiz. 103(5), 331 (2016)]
42. J.W. Shin, M.K. Cheoun, T. Kajino, A new scheme for short baseline electron antineutrino disappearance study. J. Phys. G44(9), 09LT01 (2017). arXiv:1605.00642 [physics.ins-det]

43. Y.S. Lutostansky, V.I. Lyashuk, Powerful dynamical neutrino source with a hard spectrum. Phys. At. Nucl. 63, 1288 (2000) [Yad. Fiz. 63, 1361 (2000)]

44. L.A. Mikaelian, P.E. Spivak, V.G. Tsinoev, The proposal of experiments on low energy antineutrino physics. Nucl. Phys. 70, 574 (1965)

45. A. Bungau et al., Proposal for an electron antineutrino disappearance search using high-rate ${ }^{8} \mathrm{Li}$ production and decay. Phys. Rev. Lett. 109, 141802 (2012). arXiv:1205.4419 [hep-ex]

46. F. An et al. (JUNO Collaboration), Neutrino physics with JUNO. J. Phys. G 43(3), 030401 (2016). arXiv:1507.05613 [physics.ins-det]

47. M. Abs et al., IsoDAR@ KamLAND: a conceptual design report for the technical facility. arXiv:1511.05130 [physics.acc-ph] (unpublished)

48. W.F. Hornyak, T. Lauritsen, The beta-decay of B12 and Li8. Phys. Rev. 77, 160 (1950)

49. F. Ajzenberg-Selove, Energy levels of light nuclei $A=5-10$. Nucl. Phys. A 320, 1 (1979)

50. M.G. Sternberg et al., Limit on tensor currents from Li8 $\beta$ decay. Phys. Rev. Lett. 115(18), 182501 (2015)

51. W.L. Zhan, Accelerator driven sustainable fission energy. Talk given at IPAC 2016, Busan, South Korea, May 8-13, 2016

52. V.I. Lyashuk, Y.S. Lutostansky, Reactor neutrons-antineutrino converter on the basis of lithium compounds and their solutions. Sov. At. Energy 69, 696 (1990)

53. V.F. Sears, Neutron scattering lengths and cross sections. Neutron News 3, 26 (1992)

54. These densities were taken from the density of the Centers for Disease Control and Prevention. http://www.cdc.gov

55. D.T. Ingersoll, E.J. Parma, C.W. Forsberg, J.P. Renier, Core physics characteristics and issues for the advanced high temperature reactor (AHTR). in Proceedings of ARWIF 2005, Oak Ridge National Laboratory, Feb 16-18, 2005

56. G. Battistoni, S. Muraro, P.R. Sala, F. Cerutti, A. Ferrari, S. Roesler, A. Fassò, J. Ranft, in Proceedings of the Hadronic Shower Simulation Workshop 2006, Fermilab 6-8 September 2006, ed. by M. Albrow, R. Raja. AIP Conf Proceedings, vol. 896 (2007), p. 31

57. A. Ferrari, P.R. Sala, A. Fassò, J. Ranft, FLUKA: a multi-particle transport code. CERN-2005-10, INFN/TC_05/11, SLAC-R-773 (2005)

58. S. Agostinelli et al. (GEANT4 Collaboration), GEANT4: a simulation toolkit. Nucl. Instrum. Methods A 506, 250 (2003)

59. V.I. Lyashuk, Y.S. Lutostansky, Intensive neutrino source on the base of lithium converter. arXiv:1503.01280 [physics.ins-det] (unpublished)

60. I. Tilquin et al., Experimental measurements of neutron fluxes produced by proton beams $(23-80 \mathrm{MeV})$ on $\mathrm{Be}$ and $\mathrm{Pb}$ targets. Nucl. Instrum. Methods A 545, 339 (2005)

61. M. Osipenko et al., Measurement of neutron yield by $62 \mathrm{MeV}$ proton beam on a thick Beryllium target. Nucl. Instrum. Methods A 723, 8 (2013). arXiv:1302.7226 [nucl-ex]

62. X. Zhang et al., Neutron production for $250 \mathrm{MeV}$ protons bombarding on thick grain-made tungsten target. Eur. Phys. J. A 51(8), $106(2015)$

63. H. Kudo, The rates of thermal decomposition of $\mathrm{LiOH}(\mathrm{s}), \mathrm{LiOD}(\mathrm{s})$ and LiOT(s). J. Nucl. Mater. 87, 185 (1979) 